\title{
Correction to: ACC/AATS/AHA/ASE/ASNC/ SCAI/SCCT/STS 2017 Appropriate Use Criteria for Coronary Revascularization in Patients With Stable Ischemic Heart Disease
}

\author{
Manesh R. Patel, MD, FACC, FAHA, FSCAI, ${ }^{\mathrm{a} J o h n ~ H . ~ C a l h o o n, ~ M D, ~}{ }^{\mathrm{b}}$ \\ Gregory J. Dehmer, MD, MACC, MSCAI, FAHA, ${ }^{\mathrm{c}, \mathrm{d}}$ James Aaron Grantham, MD, \\ FACC, ${ }^{\text {e,f }}$ Thomas M. Maddox, MD, MSc, FACC, FAHA, ${ }^{\text {g,h }}$ David J. Maron, MD, \\ FACC, FAHA, ${ }^{\mathrm{i}}$ and Peter K. Smith, MD, FACC ${ }^{\mathrm{j}}$ \\ a Duke University Health System, Duke Clinical Research Institute, Durham, NC \\ b Department of Cardiothoracic Surgery, Heart and Vascular Institute, University of Texas Health \\ Science Center at San Antonio, San Antonio, TX \\ c Baylor Scott \& White - Temple Memorial, Temple, TX \\ d Health Science Center, Texas A\&M University, Bryan, TX \\ e Saint Luke's Hospital, Kansas City, MO \\ f Kansas City School of Medicine, University of Missouri, Kansas City, MO \\ g VA Eastern Colorado Health Care System, Denver, CO \\ h University of Colorado, Aurora, CO \\ i Stanford University School of Medicine, Stanford, CA \\ j Division of Cardiovascular and Thoracic Surgery, Duke University, Durham, NC
}

doi: $10.1007 / \mathrm{s} 12350-018-1292-x$

\section{CORRECTION TO: \\ J NUCL CARDIOL (2017) 24:1759-1792 \\ HTTPS://DOI.ORG/10.1007/S12350-017-0917-9}

To more clearly reflect the relationship between iFR (instantaneous wave-free ratio) and FFR (fractional flow reserve), this Correction document highlights the following changes to the original document published in the Journal of Nuclear Cardiology; the version available at $J A C C^{1}$ has been updated to reflect the changes, with $J A C C$ 's Correction document available at. ${ }^{2}$

1. Page 1764, the following sentence has been added to the end of the final paragraph of "Methods": "Other physiologic measurements using the ratio of distal coronary to aortic pressure without hyperemia exist, and correlate with FFR, but are not as well-

The original article can be found online at https://doi.org/10.1007/ s12350-017-0917-9.

Reprint requests: Manesh R. Patel, MD, FACC, FAHA, FSCAI, Duke University Health System, Duke Clinical Research Institute, Durham, NC; manesh.patel@duke.edu

J Nucl Cardiol 2018;25:2191-2.

$1071-3581 / \$ 34.00$

Copyright (C) 2018 American Society of Nuclear Cardiology. validated and lack the clinical outcomes data existing for FFR.',

2. Page 1764, “Assumptions”, “General Assumptions". Assumption 1 originally comprised the following sentence: "When available, each clinical scenario includes the patient's clinical status/symptom complex, ischemic burden as determined by noninvasive functional testing, burden of coronary atherosclerosis as determined by angiography, and additional invasive testing evaluations by invasive physiology (e.g., FFR, instantaneous wave-free ratio) or intravascular imaging." The parenthetical text in that sentence has been amended to read "(e.g., FFR or other physiological pressure measurements not requiring hyperemia)',.

3. Page 1765, "Assumptions", "General Assumptions". The following sentence has been added to the end of assumption 6: "FFR is the reference standard for invasively assessing the physiological significance of a coronary artery stenosis before PCI. Newer physiological measurements that do not require hyperemia measure the ratio of distal coronary pressure to aortic pressure during the whole cardiac cycle or the wave-free portion of the cycle. Both indices have similar diagnostic concordance with FFR but have different normal 
values and have not been as well-studied as FFR. Substitution of one of the newer physiological measurements for FFR may be considered provided the appropriate reference values are used."

4. Page 1771, Table 3, "One-Vessel Disease". The footnote originally read, "*iFR measurements with appropriate normal range may be substituted for FFR." The footnote should read, "*Substitution of a newer coronary pressure ratio that does not require hyperemia for FFR may be considered provided the appropriate reference values are used.' The following item should be removed from the list of abbreviations beneath the table: "iFR, instant wave-free ratio". The same corrections should be made to Table 4, "TwoVessel Disease", Table 7, "IMA to LAD Patent and Without Significant Stenoses", and Table 8, "IMA to LAD Not Patent'.

5. Page 1775, "Discussion". Paragraph 2 contains a sentence which reads, "Fourth, the scenarios expand the use of intracoronary physiological testing, mainly with FFR." This sentence should instead read, "Fourth, the scenarios expand the use of intracoronary physiological testing, which should be performed primarily with FFR as it is a well-validated measurement and is associated with clinical outcomes following PCI."

\section{References}

1. Coronary Revascularization Writing Group, Patel Manesh R, Calhoon John H, Dehmer Gregory J, Grantham James Aaron, Maddox Thomas M, Maron David J, Smith Peter K. ACC/AATS/ AHA/ASE/ASNC/SCAI/SCCT/STS 2017 Appropriate Use Criteria for Coronary Revascularization in Patients With Stable Ischemic Heart Disease. J Am Coll Cardiol. 2017. https://doi.org/10.1016/j. jacc.2017.02.001.

2. Coronary Revascularization Writing Group, Patel Manesh R, Calhoon John H, Dehmer Gregory J, Grantham James Aaron, Maddox Thomas M, Maron David J, Smith Peter K. Correction to: ACC/AATS/AHA/ASE/ASNC/SCAI/SCCT/STS Appropriate Use Criteria for Coronary Revascularization in Patients With Stable Ischemic Heart Disease. J Am Coll Cardiol. 2017. https://doi.org/10. 1016/j.jacc.2018.03.453. 